\title{
The Effect of Widespread Pain on Knee Pain Worsening, Incident Knee Osteoarthritis and Incident Knee Pain: The Multicenter Osteoarthritis (MOST) Study
}

\author{
Lisa C. Carlesso, BSc PT, MSc. PT ${ }^{1}$ [PhD Assistant Professor], Jingbo Niu, DSc. ${ }^{2}$, Neil A. \\ Segal, MD, MS. ${ }^{3}$, Laura A. Frey Law, MPT, MS ${ }^{4}$ [PhD Associate Professor], Cora E. Lewis, \\ MD, MSPH ${ }^{5}$, Michael C. Nevitt, PhD $^{6}$ [Adjunct Professor], and Tuhina Neogi, MD, PhD ${ }^{7}$ \\ [Associate Professor] \\ ${ }^{1}$ School of Rehabilitation, Faculty of Medicine, Université de Montréal, Hôpital Maisonneuve \\ Rosemont Research Institute, Montreal, Canada \\ ${ }^{2}$ Boston University School of Medicine, Boston, MA, USA \\ ${ }^{3}$ Department of Rehabilitation Medicine, University of Kansas Medical Center, Kansas City, KS, \\ USA \\ ${ }^{4}$ Department of Physical Therapy and Rehabilitation Science, College of Medicine, University of \\ lowa at lowa City, IA, USA \\ ${ }^{5}$ Division of Preventive Medicine, University of Alabama at Birmingham, School of Medicine, \\ Birmingham, $A B$, USA \\ ${ }^{6}$ Department of Epidemiology and Biostatistics, University of California at San Francisco, San \\ Francisco, CA, USA \\ ${ }^{7}$ Boston University School of Medicine, Clinical Epidemiology Research and Training Unit, Boston \\ University, Boston, MA, USA
}

\section{Abstract}

Objectives-Whether widespread pain (WSP) affects the risk of developing knee pain or knee osteoarthritis (OA) is unknown and could enhance understanding of pain mechanisms in OA.

Methods-Subjects from the Multicenter Osteoarthritis (MOST) study, a NIH-funded prospective cohort of older adults with or at risk of knee OA were characterized with regards to WSP, defined as pain above and below the waist, on both sides of the body and axially, using a standard homunculus, excluding knee pain at 60 months (baseline). Follow up occurred 2 years later. We assessed the relation of WSP to odds of knee pain worsening ( 2 -point increase in the WOMAC pain subscale) using logistic regression and to odds of incident radiographic knee OA (ROA) (Kellgren \& Lawrence $\geq$ grade 2 of either knee among those free of ROA at baseline) and incident consistent frequent knee pain (CFKP) (knee pain on most days during the past month

Requests for reprints and correspondence to: Lisa C Carlesso, École de réadaptation, Faculté de médecine, Université de Montréal, C.P. 6128, succursale Centre-ville, Montréal (Québec) Canada H3C 3J7. 
among participants free of knee pain at baseline) in one or both knees using multinomial regression adjusting for potential confounders.

Results-There were 1752 participants available for analysis (mean age (SD) 67.0 (7.7); BMI $30.5(5.9) \mathrm{kg} / \mathrm{m}^{2} ; 59 \%$ women). Baseline presence of WSP was not associated with worsened knee pain [adjusted OR 1.15, (0.89-1.48), $\mathrm{p}=0.30$ ], ROA [adjusted OR 0.86, $(0.46-1.63) \mathrm{p}=0.65$ ] or incident CFKP [adjusted OR 1.69, (0.96-2.96), $\mathrm{p}=0.07$ ].

Conclusion-WSP was not significantly associated with worsening knee pain, incident ROA or CFKP. Development of knee pain and ROA does not appear to be influenced by underlying WSP.

\section{Keywords}

widespread pain; knee pain; osteoarthritis

\section{Introduction}

Osteoarthritis (OA) of the knee is one of the most common musculoskeletal pain disorders and is a leading cause of disability(1). The prevalence of knee OA is $37 \%$ in people aged $\geq$ 45 years $(2,3)$ and it is symptomatic in up to $17 \%$ of those with radiological evidence of OA(3). Despite its prevalence and burden, the etiology of OA pain is not well understood. Some studies have suggested that widespread pain (WSP) may be associated with increased pain and decreased function(4), in those living with knee OA, $(5,6)$ and more generally contributes to poor mental and physical functioning,(7) morbidity,(8) and mortality(9).

The tendency of pain to become widespread is thought to be the result of elevated sensitivity of the central nervous system,(10) associated with increasing intensity and duration of pain(11). Dysfunction in the central processing of pain can occur through the alterations of both ascending and descending pathways resulting in diffuse hyperalgesia and/or allodynia, thus potentiating the onset or increased severity of knee pain(12). We have recently shown that in people with or at risk of knee OA, knee pain severity is predictive of the development of WSP two years later(13). However, it is unknown whether a bidirectional relationship may exist. The literature examining the association between WSP and knee pain has been largely cross-sectional. WSP was associated with greater prevalence of consistent versus inconsistent knee pain in one study,(6) and others have shown a stronger association between WSP and knee pain with increasing structural severity of OA(14), as well as an association of WSP with a combination of high knee pain severity and low grade structural severity of OA(15). One longitudinal study by Jinks et al.(16) found an association of multijoint pain ( $\geq 2$ joints) with incident knee pain. However these studies did not adjust for important potential confounders, such as pain catastrophizing,(17) quality of sleep,(18) or fatigue(19) and varying definitions of WSP were used, making direct comparisons difficult.

Regardless of the potential for WSP to impact development of knee pain, it is also important to understand whether WSP can contribute to the development of structural pathology. The literature base regarding the association of WSP with incident knee OA is lacking and evidence regarding potential mechanisms that may account for the relationship are conflicting and complex. For example, WSP is known to have a negative impact on physical 
activity(20). In a longitudinal study, McBeth et al(20). reported that those with WSP were twice as likely to have lower levels of physical activity or to be engaging in less than 20 minutes of exercise in the past month at follow-up more than 2 years later(20). As physical activity helps promote cartilage volume while limiting the occurrence of cartilage defects (in the absence of knee injury)(21), it is thought that this may decrease the risk of knee OA. This would suggest that people with WSP may have an increased risk of developing incident knee OA due to lower levels of physical activity. However, studies have reported varying results of physical activity on incident knee OA including no effect, $(22,23)$ a protective effect(24) or an increased risk(25). Alternatively, it is speculated that WSP may lead individuals to limit excessive joint loading, which could potentially be beneficial. Slower walking speeds are associated with lower knee joint and ground reaction forces(26) and faster walking speeds are associated with increased knee joint loading,(27) which in theory, suggest that slower walking may lead to a lower incidence of knee OA. However, one longitudinal study presented results contrary to this hypothesis by demonstrating that people with faster walking speeds had a lower incident of knee OA(28). In a study comparing the impact of pain location versus pain severity on lower extremity physical function,

Eggermont et al.(29) reported a significant trend in walking speeds, with those having WSP being the slowest versus those with multisite, single site or no pain. Given these conflicting results and the limited evidence of the association of WSP with incident knee OA, clarification of the association of WSP with incident knee OA is warranted.

Understanding whether having WSP can lead to new onset or worsening of knee pain or the development of knee OA structural pathology is valuable as it can lead to more timely targeted interventions for pain management that may help mitigate the detrimental effects of WSP on knee pain or knee OA. We therefore examined the longitudinal relation of WSP to worsening of knee pain severity, incident knee pain, and ROA in a large prospective cohort.

\section{Materials and Methods}

\section{Study sample}

The Multicenter Osteoarthritis Study is a NIH funded, longitudinal cohort that initially enrolled 3026 community dwelling adults between the ages of 50-79 years who had or were at risk of developing knee OA. Subjects were recruited from two sites- Birmingham, Alabama and Iowa City, Iowa, and were assessed at 0-month, 30-month, 60-month and 84month study visits. Initial recruitment occurred between 2003-2005. Details of the cohort have been published elsewhere(30). The study protocol was approved by the institutional review boards at the University of Iowa, University of Alabama at Birmingham, University of California at San Francisco and Boston University Medical Center. All participants gave written informed consent. The current sample comprised participants who attended the 60month visit (baseline for this study as this was the first time that several confounding variables measured) and who attended the follow-up visit at 84 months.

\section{Exposure: Widespread pain}

The definition of WSP was operationalized as requiring pain to be present in each of the following regions - above (1) and below (2) the waist, on both sides of the body (right (3) 
and left (4)) and axially (5), using participants' pain drawings on a validated standard homunculus(31). Given our focus on knee OA and knee pain, we did not include knee pain in the definition of WSP (i.e., other lower extremity pain was required). Therefore, participants had to have at least 3 painful joints to be classified as having WSP. Those who met the definition were classified as having WSP and those who did not were classified as not having WSP.

\section{Outcomes: Worsening knee pain, consistent frequent knee pain, and radiographic osteoarthritis}

Knee pain severity was assessed at each study visit using the pain subscale (scores range from 0-20) of the valid and reliable Western Ontario and McMaster Universities Arthritis Index (WOMAC) (Likert version) (32). Participants were classified as having worsening of knee pain if they reported an increase in pain of $\geq$ two points in either knee at 84 months compared to baseline knee pain at 60 months. For the WOMAC pain subscale, a two-point change has been shown to be the minimal clinically important difference(33).

Frequent knee pain was assessed at each study visit. Consistent frequent knee pain (CFKP) was defined as a participant answering 'yes' to having knee pain on most days during the past month at the telephone screen one month prior to the clinic visit and again at the clinic visit. If either knee met the definition for CFKP, that participant was considered to have CFKP. Incident CKFP was defined as the presence of CFKP at 84 months among those who did not have CFKP at 60 months.

Bilateral weight-bearing fixed-flexion posteroanterior radiographs and lateral weight-bearing radiographs of the knee were obtained at each study visit. Radiographic severity in the tibiofemoral joint was graded by two experienced readers blinded to clinical data according to Kellgren and Lawrence (KL) criteria (0-4)(34),(35) Any disagreements between readers were adjudicated by a third reader along with the first 2 readers to reach consensus. The inter-rater reliability weighted kappa for the KL grade was 0.80 . A participant was defined as having incident radiographic OA (ROA) based on the presence of tibiofemoral OA, KL grade $\geq 2$, at 84 months among those who did not have ROA at the 60-month clinic visit (baseline for this study).

\section{Potential confounders}

Potential confounders included age, sex, body mass index (BMI), comorbidities, depressive symptoms, pain catastrophizing, pain severity, physical activity, sleep, fatigue and study site were collected at baseline (60 months). Body mass index (BMI, $\mathrm{kg} / \mathrm{m}^{2}$ ) was calculated (weight in kilograms divided by the squared height in meters) from measurements taken by trained and certified staff at the 60-month clinic visit. Participants completed a modified version of the Charlson Comorbidity Index, a validated, self-reported measure of comorbid conditions(36). The Center for Epidemiologic Studies Depression Scale (CES-D) was utilized as a measure of depressive symptoms(37). A score of 16 or greater has been used as an indicator of depressive symptoms(37). Pain catastrophizing was measured using one item from the Coping Strategies Questionnaire. Use of the brief one-item scale has been shown to be valid and reliable(38). The item is scored between $0-6$ with higher scores indicating 
greater frequency of the behaviour. Pain catastrophizing was defined as being present if the score was $\geq 1$. Knee pain severity was measured using the Western Ontario and McMaster Universities Osteoarthritis Index (WOMAC; Likert version) pain subscale (0-20 score range) on a knee-specific basis(32). Higher scores on the WOMAC indicate greater pain. The higher of the two ratings was used to indicate worst knee pain in each subject. The Physical Activity Scale for the Elderly (PASE) is a validated measure consisting of 20 items asking about frequency of each activity(39). These responses are then transformed according to New England Research Institute's validated regression equations to achieve a total activity score. Sleep was assessed with the following question: "During the past 7 days, how would you rate your sleep quality overall?" with responses ranging from very bad to very good on a 4-point Likert scale. Those indicating "bad" or "very bad" sleep were classified as having bad sleep. Fatigue was measured on a numeric rating scale (0-10) using the following question: "During the past 7 days, what number between 0 and 10 best describes your usual level of fatigue?" Higher numbers indicated a higher level of fatigue.

\section{Statistical analyses}

We evaluated the association between WSP and worsening knee pain using logistic regression. We also examined the relation of WSP to bilateral and unilateral involvement for each of incident ROA and incident CFKP compared with neither knee having the condition using multinomial regression. Those who had a knee replacement over the course of the study were considered to have experienced the outcomes of worsening pain severity, CFKP, and were considered to have incident ROA. All models were adjusted for age, sex, BMI, comorbidities, physical activity and clinic site, while the model was also adjusted for depressive symptoms, pain catastrophizing, fatigue, sleep and ROA when worsening knee pain and CFKP were the outcome, and for WOMAC pain severity when ROA was the outcome. We also conducted a sensitivity analysis to evaluate the effect of WSP from the parent study baseline (0 months) on worsening knee pain, incident CFKP, and incident ROA at 84 months, with similar adjustment of potential confounders with the exception of pain catastrophizing, sleep and fatigue as these variables were first collected at 60 months. The sensitivity analyses were conducted to improve precision by assessing greater endpoints over a longer period of time. All analyses were performed using SAS 9.3 (SAS Institute, Gary, North Carolina, USA).

\section{Ethics}

The procedures followed were in accordance with the ethical standards of the responsible committee on human experimentation and with the Helsinki Declaration of 1975, as revised in 2000. All subjects gave informed consent to participate in this research. Approval for this secondary analysis was granted by the Boston University Medical Center IRB \# was H-27296.

\section{Results}

There were 1752 subjects eligible for worsened knee pain (mean age (SD); 67.0 (7.7); BMI $30.5(5.9) \mathrm{kg} / \mathrm{m}^{2}, 59 \%$ women), 815 eligible subjects for analysis of incident CFKP (mean 
age (SD); 66.9 (7.4); BMI $29.5(5.2) \mathrm{kg} / \mathrm{m}^{2}, 54 \%$ women), and 675 for incident ROA (age 65.6 (7.4); BMI $28.9(4.7) \mathrm{kg} / \mathrm{m}^{2}, 60 \%$ women) (see Table 1).

\section{WSP and odds of worsening knee pain}

In the 549 people with WSP at baseline (60 months), 170(31.0\%) also had worsening knee pain. The presence of WSP at baseline was associated with a non-statistically significant $15 \%$ higher odds of worsening knee pain (adjusted OR 1.15 95\% CI $0.89-1.48, \mathrm{p}=0.30$ ) at 84 months compared to those without WSP at baseline.

\section{WSP and odds of CFKP}

The baseline presence of WSP was associated with a non-statistically significant 69\% increased odds of incident CFKP 2 years later compared with those without WSP (adjusted OR $1.69,95 \%$ CI $0.96-2.96, \mathrm{p}=0.07$ ). WSP was also associated with non-statistically significant increased odds of incident unilateral and bilateral CFKP (see Table 2).

\section{WSP and odds of ROA}

Baseline WSP was associated with a non-statistically significant $14 \%$ lower odds of incident ROA, (adjusted OR $0.86,95 \%$ CI $0.46-1.63, \mathrm{p}=0.65$ ). There was not a statistically significant association between WSP and either incident unilateral or bilateral ROA, compared with no ROA (see Table 2).

\section{Sensitivity Analyses}

As with our primary analyses, the findings for knee pain worsening and the incident ROA groups (data not shown) were similar. However, WSP at 0 months was significantly associated with approximately double the odds of incident CFKP 7 years later compared with those who did not have CFKP [adjusted OR $2.05(1.35,3.13) \mathrm{p}=0.008$ ] and in those with unilateral CFKP compared with those without CFKP [adjusted OR $2.19(1.37,3.50)$ $\mathrm{p}=0.0001]$. The presence of WSP was associated with a non-statistically significant $73 \%$ higher incidence of bilateral CFKP. Given this result, we reran the main incident CFKP model (i.e. 60-84 months) without pain catastrophizing, fatigue and sleep. A significant association of WSP was found in those with incident CFKP [adjusted OR $1.90(1.10,3.29)$ $\mathrm{p}=0.022]$ compared to those without CFKP, indicating that pain catastrophizing, fatigue and sleep were attenuating the effect of WSP over a 2-year time frame. There were no statistically significant findings for either unilateral or bilateral incident CFKP.

\section{Discussion}

In contrast to previous largely cross-sectional studies, we found no association of WSP with knee pain, defined either as worsening of knee pain severity or incident consistent frequent knee pain after 2 years. We also demonstrated that WSP is not predictive of the development of structural pathology of OA at the knee over a 2-year window.

Our findings differ from those of some prior studies. Most of the prior studies have been cross-sectional, potentially suffering from reverse causation bias and have included subjects with both WSP and knee pain pre-existing $(14,15)$. Some have alluded to a potential dose- 
response relationship between number of painful joints and knee pain severity $(40,41)$. The one prior longitudinal study focused on multi-joint pain, defined as $\geq 2$ joints(16). Our stricter definition required at least three painful joints in addition to a painful knee joint. Thus our results, when considered with those of Jinks et al.(16) suggest that a dose-response relationship does not exist. While the longitudinal results of this study do not support the idea that WSP is involved in the development of worsening pain, incident pain or ROA, a previous longitudinal analysis in this cohort has shed some light on the sequential relationships of knee pain and knee OA to WSP demonstrating that knee pain severity is predictive of the development of WSP(13). However, when the results of our sensitivity analysis are considered, the notion that the relationship between these variables may be bidirectional in certain circumstances cannot be excluded.

Although we found no association longitudinally, with longer duration of follow-up, there was a suggestion of increased odds of consistent frequent knee pain. However, comparable sensitivity analyses (i.e. important confounders removed) conducted between $60-84$ months demonstrated that the confounders attenuated the effect of WSP on consistent frequent knee pain and may explain in part, why prior cross-sectional studies, which also did not adjust for those factors, were positive. However, our analyses cannot confirm the precise role of pain catastrophizing, sleep quality or fatigue as confounders or mediators. For example, it is possible that WSP could cause any of these variables, which in turn could lead to incident CFKP. Given the 5-year difference in time frames, and inability to adjust for confounders since study baseline, we cannot rule out the possibility that duration of WSP may have had an effect. We are unaware of any other studies that have prospectively studied the relationship between WSP and incident ROA. While our findings in this regard were nonsignificant, the direction of the effect estimates suggests that WSP may possibly have a protective effect on the development of ROA. This would support the hypothesis that WSP may lead to lower loads transmitted to the knee, such as through decreased walking speed and/or other strategies employed by those with WSP. At this juncture, such interpretation is speculative.

Our study has several strengths and limitations to consider. The prospective nature of our investigation in this population provides the first longitudinal evidence regarding the association of WSP (defined in a validated manner) on worsening knee pain, incident knee pain, and incident knee ROA. We were able to adjust for important confounders; however, the possibility of residual confounding remains as with any observational study. We did not consider an intermediate stage of regional pain (i.e., those with multi-joint pain but not meeting the WSP criteria). As previous work has reported an association of multi-joint pain (i.e. $\geq 2$ joints) and incident knee pain, we cannot rule out that this association may exist in our cohort and may have diminished our ability to detect a difference relative to those with no other joint pain. Lastly, our primary analyses only considered a 2-year follow-up. The influence of WSP on worsening knee pain, incident knee pain, and incident knee ROA may require a longer follow- up to delineate its influence more clearly, as is suggested by the secondary analyses. However, that no significant influence was observed over a 2-year window suggests a relatively weak effect of WSP on knee OA overall. 
In conclusion, we found no association between WSP and worsening knee pain, incident knee pain, or incident knee ROA over 24 months. The current study suggests that the effect of pain sensitivity associated with WSP itself may not necessarily lead to the worsening or onset of knee pain or knee structural pathology. The results of our sensitivity analyses provide an opportunity for future studies to clarify the role of pain catastrophizing, sleep quality or fatigue as confounders or mediators in this relationship.

\section{Acknowledgments}

LC was supported by a Fellowship from the Canadian Institutes of Health Research and a Collaborative scholarship from Osteoarthritis Research Society International. Dr. Neogi's work is supported by NIH grants P60-AR-47785 and R01-AR-062506 for this work. The Multicenter Osteoarthritis Study is supported by NIH grants U01AG-18820, U01-AG-18832, U01-AG-18947, and U01-AG-19079.

\section{References}

1. Guccione AA, Felson DT, Anderson JJ, Anthony JM, Zhang Y, Wilson PW, et al. The effects of specific medical conditions on the functional limitations of elders in the Framingham Study. Am J Public Health. 1994; 84:351-358. [PubMed: 8129049]

2. Dillon CF, Rasch EK, Gu Q, Hirsch R. Prevalence of knee osteoarthritis in the United States: arthritis data from the Third National Health and Nutrition Examination Survey 1991-94. J Rheumatol. 2006; 33:2271-2279. [PubMed: 17013996]

3. Jordan JM, Helmick CG, Renner JB, Luta G, Dragomir AD, Woodard J, et al. Prevalence of knee symptoms and radiographic and symptomatic knee osteoarthritis in African Americans and Caucasians: the Johnston County Osteoarthritis Project. J Rheumatol. 2007; 34:172-180. [PubMed: 17216685]

4. McBeth J, Lacey RJ, Wilkie R. Predictors of new-onset widespread pain in older adults: results from a population-based prospective cohort study in the UK. Arthritis \&amp; rheumatology (Hoboken, NJ). 2014; 66:757-767.

5. White DK, Felson DT, Niu J, Nevitt MC, Lewis CE, Torner JC, et al. Reasons for functional decline despite reductions in knee pain: the Multicenter Osteoarthritis Study. Phys Ther. 2011; 91:18491856. [PubMed: 22003168]

6. Neogi T, Nevitt M, Yang M, Curtis J, Torner J, Felson D. Consistency of Knee Pain: Correlates and Association with Function. Osteoarthritis Cartilage. 2010 Oct 18.:1250-1255. [PubMed: 20708003]

7. Lacey RJ, Belcher J, Rathod T, Wilkie R, Thomas E, McBeth J. Pain at multiple body sites and health-related quality of life in older adults: results from the North Staffordshire Osteoarthritis Project. Rheumatology (Oxford). 2014; 53:2071-2079. [PubMed: 24925881]

8. Kadam UT, Thomas E, Croft PR. Is chronic widespread pain a predictor of all-cause morbidity? A 3 year prospective population based study in family practice. J Rheumatol. 2005; 32:1341-1348. [PubMed: 15996075]

9. Nitter A, Forseth K. Mortality rate and causes of death in women with self-reported musculoskeletal pain: Results from a 17-year follow-up study. Scand J Pain. 2013:86-92.

10. Latremoliere A, Woolf CJ. Central sensitization: a generator of pain hypersensitivity by central neural plasticity. J Pain. 2009; 10:895-926. [PubMed: 19712899]

11. Arendt-Nielsen L, Graven-Nielsen T. Translational musculoskeletal pain research. Best Practice \& Research Clinical Rheumatology. 2011; 25:209-226. [PubMed: 22094197]

12. Fingleton C, Smart K, Moloney N, Fullen BM, Doody C. Pain sensitization in people with knee osteoarthritis: a systematic review and meta-analysis. Osteoarthritis Cartilage. 2015; 23:10431056. [PubMed: 25749012]

13. Carlesso LC, Segal N, Curtis JR, Wise BL, Law LF, Nevitt M, et al. Knee Pain Severity Rather Than Structural Damage is a Risk Factor for Incident Widespread Pain: The Multicenter Osteoarthritis MOST) Study. Arthritis Care Res (Hoboken). 2016 Accepted Article. 
14. Schiphof D, Kerkhof HJ, Damen J, de Klerk BM, Hofman A, Koes BW, et al. Factors for pain in patients with different grades of knee osteoarthritis. Arthritis Care Res (Hoboken). 2013; 65:695702. [PubMed: 23203619]

15. Riddle DL, Stratford PW. Knee pain during daily tasks, knee osteoarthritis severity, and widespread pain. Phys Ther. 2014; 94:490-498. [PubMed: 24231230]

16. Jinks C, Jordan KP, Blagojevic M, Croft P. Predictors of onset and progression of knee pain in adults living in the community. A prospective study. Rheumatology (Oxford). 2008; 47:368-374. [PubMed: 18263594]

17. Giesecke T, Williams DA, Harris RE, Cupps TR, Tian X, Tian TX, et al. Subgrouping of fibromyalgia patients on the basis of pressure-pain thresholds and psychological factors. Arthritis Rheum. 2003; 48:2916-2922. [PubMed: 14558098]

18. McBeth J, Wilkie R, Bedson J, Chew-Graham C, Lacey RJ. Sleep disturbance and chronic widespread pain. Curr Rheumatol Rep. 2015; 17:469. [PubMed: 25604572]

19. Wolfe F, Hawley DJ, Wilson K. The prevalence and meaning of fatigue in rheumatic disease. J Rheumatol. 1996; 23:1407-1417. [PubMed: 8856621]

20. McBeth J, Nicholl BI, Cordingley L, Davies KA, Macfarlane GJ. Chronic widespread pain predicts physical inactivity: Results from the prospective EPIFUND study. European Journal of Pain. 2010; 14:972-979. [PubMed: 20400346]

21. Urquhart D, Tobing J, Hanna F, Berry P, Wluka A, Ding C, et al. What is the effect of physical activity on the knee joint? A systematic review. Med Sci Sports Exerc. 2011 Mar.(9):432-442. [PubMed: 20631641]

22. Felson DT, Niu J, Clancy M, Sack B, Aliabadi P, Zhang Y. Effect of recreational physical activities on the development of knee osteoarthritis in older adults of different weights: the Framingham Study. Arthritis Rheum. 2007; 57:6-12. [PubMed: 17266077]

23. Mork PJ, Holtermann A, Nilsen TI. Effect of body mass index and physical exercise on risk of knee and hip osteoarthritis: longitudinal data from the Norwegian HUNT Study. J Epidemiol Community Health. 2012; 66:678-683. [PubMed: 22511797]

24. Hart DJ, Doyle DV, Spector TD. Incidence and risk factors for radiographic knee osteoarthritis in middle-aged women: the Chingford Study. Arthritis Rheum. 1999; 42:17-24. [PubMed: 9920009]

25. Felson DT, Zhang Y, Hannan MT, Naimark A, Weissman B, Aliabadi P, et al. Risk factors for incident radiographic knee osteoarthritis in the elderly: the Framingham Study. Arthritis Rheum. 1997; 40:728-733. [PubMed: 9125257]

26. Zeni J Jr, Higginson J. Differences in gait parameters between healthy subjects and persons with moderate and severe knee osteoarthritis: a result of altered walking speed? Clin Biomech (Bristol, Avon). 2009 May.24:372-378.

27. Teixeira-Salmela LF, Nadeau S, Milot MH, Gravel D, Requiao LF. Effects of cadence on energy generation and absorption at lower extremity joints during gait. Clin Biomech (Bristol, Avon). $2008 ; 23: 769-778$.

28. Purser JL, Golightly YM, Feng Q, Helmick CG, Renner JB, Jordan JM. Association of slower walking speed with incident knee osteoarthritis-related outcomes. Arthritis Care Res (Hoboken). 2012; 64:1028-1035. [PubMed: 22392700]

29. Eggermont LH, Bean JF, Guralnik JM, Leveille SG. Comparing pain severity versus pain location in the MOBILIZE Boston study: chronic pain and lower extremity function. J Gerontol A Biol Sci Med Sci. 2009; 64:763-770. [PubMed: 19228782]

30. Segal NA, Nevitt MC, Gross KD, Hietpas J, Glass NA, Lewis CE, et al. The Multicenter Osteoarthritis Study: opportunities for rehabilitation research. Pm r. 2013; 5:647-654. [PubMed: 23953013]

31. Leveille SG, Zhang Y, McMullen W, Kelly-Hayes M, Felson DT. Sex Differences in musculoskeletal pain in older adults. Pain. 2005; 116:332-338. [PubMed: 15982814]

32. Bellamy N, Buchanan WW, Goldsmith CH, Campbell J, Stitt LW. Validation study of WOMAC: a health status instrument for measuring clinically important patient relevant outcomes to antirheumatic drug therapy in patients with osteoarthritis of the hip or knee. J Rheumatol. 1988; 15:1833-1840. [PubMed: 3068365] 
33. Angst F, Aeschlimann A, Stucki G. Smallest detectable and minimal clinically important differences of rehabilitation intervention with their implications for required sample sizes using WOMAC and SF-36 quality of life measurement instruments in patients with osteoarthritis of the lower extremities. Arthritis Rheum. 2001; 45:384-91. [PubMed: 11501727]

34. Kellgren JH, Lawrence JS. Radiological assessment of osteo-arthrosis. Ann Rheum Dis. 1957; 16:494-502. [PubMed: 13498604]

35. Altman RD, Hochberg M, Murphy WA Jr, Wolfe F, Lequesne M. Atlas of individual radiographic features in osteoarthritis. Osteoarthritis Cartilage. 1995; 3(Suppl A):3-70. [PubMed: 8581752]

36. Charlson ME, Pompei P, Ales KL, MacKenzie CR. A new method of classifying prognostic comorbidity in longitudinal studies: Development and validation. J Chronic Dis. 1987; 40:373383. [PubMed: 3558716]

37. Radloff L. The CES-D scale: a self-report depression scale for research in the general population. Appl Psychol Meas. 1977; 1:385-401.

38. Jensen MP, Keefe FJ, Lefebvre JC, Romano JM, Turner JA. One- and two-item measures of pain beliefs and coping strategies. Pain. 2003; 104:453-469. [PubMed: 12927618]

39. Washburn RA, Smith KW, Jette AM, Janney CA. The Physical Activity Scale for the Elderly (PASE): development and evaluation. J Clin Epidemiol. 1993; 46:153-162. [PubMed: 8437031]

40. Croft P, Jordan K, Jinks C. "Pain elsewhere" and the impact of knee pain in older people. Arthritis Rheum. 2005; 52:2350-2354. [PubMed: 16052574]

41. Kamaleri Y, Natvig B, Ihlebaek C, Bruusgaard D. Localized or widespread musculoskeletal pain: does it matter? Pain. 2008 Aug 15.138:41-46. [PubMed: 18077092] 


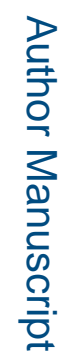

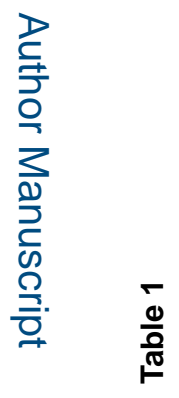

\begin{tabular}{|c|c|c|c|c|c|c|c|c|c|c|c|}
\hline 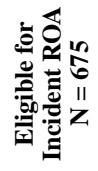 & 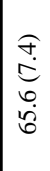 & 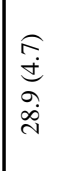 & 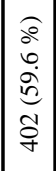 & $\widehat{\vec{e}}$ & 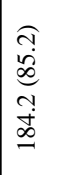 & $\begin{array}{l}\hat{\sigma} \\
\stackrel{d}{d} \\
\stackrel{i}{i}\end{array}$ & \begin{tabular}{l}
$\hat{\sigma}$ \\
$\dot{e}$ \\
\multirow{b}{b}{}
\end{tabular} & 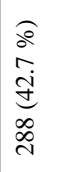 & 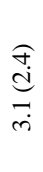 & 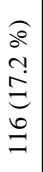 & 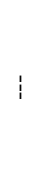 \\
\hline 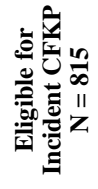 & 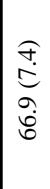 & 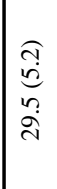 & 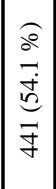 & $\hat{\overrightarrow{0}}$ & $\begin{array}{l}\sigma \\
\dot{\infty} \\
\infty \\
m \\
\stackrel{0}{0} \\
\infty\end{array}$ & 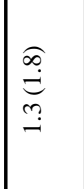 & $\begin{array}{l}\widehat{\sigma} \\
\vec{e} \\
\dot{y} \\
\dot{n}\end{array}$ & 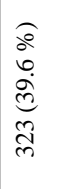 & $\begin{array}{l}\text { తิ } \\
\text { ते } \\
\text { ì }\end{array}$ & 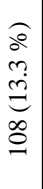 & 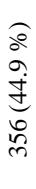 \\
\hline 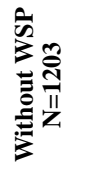 & 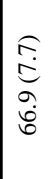 & \begin{tabular}{|l}
$\hat{\alpha}$ \\
$\dot{n}$ \\
$-\dot{0}$ \\
$\dot{n}$
\end{tabular} & $\mid \begin{array}{l}\hat{\sigma} \\
\hat{0} \\
\widehat{\sigma} \\
\end{array}$ & $\widehat{\overrightarrow{0}}$ & $\begin{array}{l}\hat{0} \\
\dot{0} \\
\stackrel{0}{0} \\
\stackrel{0}{\infty} \\
\stackrel{0}{0}\end{array}$ & 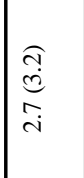 & $\begin{array}{l}\hat{\sigma} \\
\hat{\omega} \\
m \\
i \\
\dot{n}\end{array}$ & $\mid \begin{array}{l}\hat{\sigma} \\
\hat{\partial} \\
\hat{q} \\
\tilde{\sigma}\end{array}$ & $\begin{array}{l}\text { तิ } \\
\text { d } \\
\text { i }\end{array}$ & 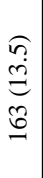 & 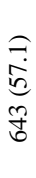 \\
\hline 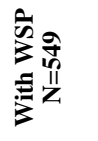 & 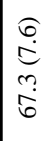 & 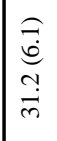 & 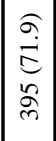 & $\begin{array}{l}\hat{\jmath} \\
\hat{e} \\
0\end{array}$ & 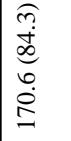 & 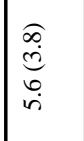 & $\begin{array}{l}\infty \\
\infty \\
\infty \\
\alpha \\
\alpha\end{array}$ & 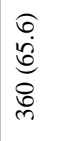 & $\begin{array}{l}\underset{f}{\stackrel{d}{d}} \\
\stackrel{m}{+}\end{array}$ & $\begin{array}{l}\widehat{\alpha} \\
\stackrel{d}{0} \\
\text { d } \\
\text { I }\end{array}$ & 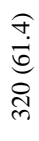 \\
\hline \multirow[t]{2}{*}{ 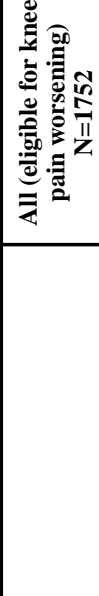 } & 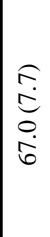 & $\begin{array}{l}\hat{\sigma} \\
\tilde{\sigma} \\
n \\
\tilde{z} \\
\tilde{n}\end{array}$ & 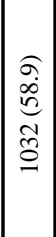 & $\widehat{\overrightarrow{0}}$ & 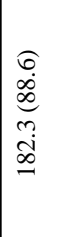 & 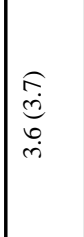 & 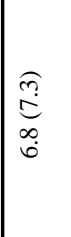 & $\mid$\begin{tabular}{c}
0 \\
0 \\
$\infty$ \\
\multirow{j}{0}{} \\
$\tilde{N}$ \\
$\infty$
\end{tabular} & 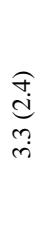 & 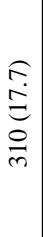 & $\begin{array}{l}\tilde{n} \\
\infty \\
0 \\
0 \\
\delta \\
\delta\end{array}$ \\
\hline & 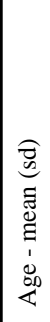 & 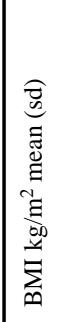 & 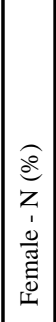 & 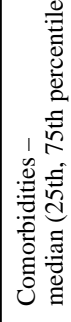 & 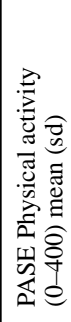 & 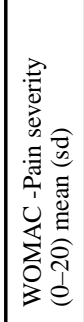 & 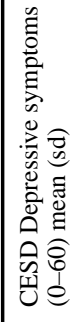 & 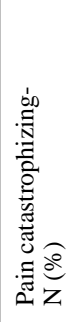 & 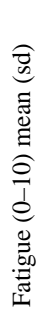 & 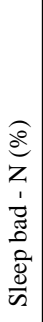 & 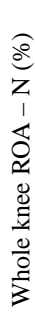 \\
\hline
\end{tabular}

J Rheumatol. Author manuscript; available in PMC 2018 April 01. 
Table 2

Risk of WSP to incident consistent frequent knee pain and ROA

\begin{tabular}{|c|c|c|c|c|}
\hline \multicolumn{5}{|c|}{ Incident consistent frequent knee pain } \\
\hline & $\begin{array}{l}\text { Risk among subjects } \\
\text { without WSP, n (\%) } \\
\text { N=669 }\end{array}$ & $\begin{array}{l}\text { Risk among subjects } \\
\text { with WSP, n (\%) } \\
\text { N=146 }\end{array}$ & $\begin{array}{c}\text { Crude OR } \\
\text { WSP vs. } \\
\text { without WSP (ref.) }\end{array}$ & $\begin{array}{c}\text { Adjusted OR* } \\
\text { WSP vs. } \\
\text { without WSP (ref.) }\end{array}$ \\
\hline $\begin{array}{l}\text { Either Knee Affected } \\
\text { (vs. None Affected) }\end{array}$ & $69(10.3)$ & $27(18.5)$ & 1.97 & $1.69(0.96,2.96) \mathrm{p}=0.07$ \\
\hline \multicolumn{5}{|c|}{ Number of Knees Affected: } \\
\hline Unilateral & $55(8.2)$ & $18(12.3)$ & 1.65 & $1.58(0.83,3.02) \mathrm{p}=0.17$ \\
\hline Bilateral & $14(2.1)$ & $9(6.2)$ & 3.24 & $1.82(0.64,5.16) \mathrm{p}=0.26$ \\
\hline \multicolumn{5}{|c|}{ Incident $R O A$} \\
\hline & $\begin{array}{l}\text { Risk among subjects } \\
\text { without WSP, } \mathrm{n}(\%) \\
\mathrm{N}=475\end{array}$ & $\begin{array}{l}\text { Risk among subjects } \\
\text { with WSP, n (\%) } \\
\text { N=200 }\end{array}$ & $\begin{array}{c}\text { Crude OR } \\
\text { WSP vs. } \\
\text { without WSP (ref.) }\end{array}$ & $\begin{array}{c}\text { Adjusted OR }{ }^{\dagger}, \\
\text { WSP vs. } \\
\text { without WSP (ref.) }\end{array}$ \\
\hline $\begin{array}{l}\text { Either Knee Affected } \\
\text { (vs. None Affected) }\end{array}$ & $43(9.1)$ & $22(11.0)$ & 1.24 & $0.86(0.46,1.63) \mathrm{p}=0.65$ \\
\hline \multicolumn{5}{|c|}{ Number of Knees Affected: } \\
\hline Unilateral & $38(8.0)$ & $21(10.5)$ & 1.34 & $0.99(0.51,1.92) \mathrm{p}=0.97$ \\
\hline Bilateral & $5(1.1)$ & $1(0.5)$ & 0.49 & $0.23(0.02,2.53) \mathrm{p}=0.23$ \\
\hline
\end{tabular}

* Adjusted for age at 60 mos, sex, BMI, comorbidities, physical activity, clinic site, ROA either knee, depressive symptoms, catastrophizing, sleep and fatigue

${ }^{\dagger}$ Adjusted for age at $60 \mathrm{mos}$, sex, BMI, comorbidities, physical activity, clinic site and knee pain severity 